\title{
High-Resolution Spectra of the 6300-Å Region of Comet P/Halley
}

\author{
Michael R. Combi \\ Space Physics Research Laboratory, University of Michigan, 2455 Hayward Street, Ann Arbor, Michigan 48109-2143 \\ AND \\ RICHARD E. MCCROSKY \\ Harvard-Smithsonian Center for Astrophysics, 60 Garden Street, Cambridge, Massachusetts 02138
}

Received November 5, 1990; revised January 2, 1991

\begin{abstract}
A series of high resolution spectra of the $6300-\AA$ region of Comet $\mathrm{P} / \mathrm{Halley}$ were taken with the echelle/reticon system on the 61-in. telescope at the Harvard-Smithsonian Oak Ridge Observatory from 23 October 1985 through 5 January 1986. Relative contributions of various rotational lines within the $(0,8,0)$ band of $\mathrm{NH}_{2}$ and of the cometary and airglow contributions of $O\left({ }^{1} D\right)$ were clearly spectrally separated. Measurements were taken both centered on the nucleus as well as offset by various displacements in different directions. A number of important implications have resulted from the analysis of these data. (1) The relative fluxes of different rotational members of the $\mathrm{NH}_{2}(0,8,0)$ band vary not only from dayto-day, as previously reported, but also with radial distance within the coma. Indications are that the vib-rotational structure of the band could be useful as a diagnostic for coma temperatures and/ or outflow kinematics. (2) Deviations from circular symmetry were negligible for $\mathrm{NH}_{2}$ but detectable for $\mathrm{O}\left({ }^{1} \mathrm{D}\right)$, having only a small $(0-10 \%)$ average sunward asymmetry. (3) The inner radial brightness distribution for $O\left({ }^{1} D\right)$ is consistent with its production from the photodissociation of its parent $\left(\mathrm{H}_{2} \mathrm{O}\right)$. (4) The inner radial brightness distributions for $\mathrm{NH}_{2}$ were reasonably consistent with previously published results found for Halley and other comets. (5) Some inconsistencies were found in the comparison of the cometary $\mathrm{NH}_{2}$ line wavelengths with the standard laboratory values. (c) 1991 Academic Press, Inc.
\end{abstract}

\section{INTRODUCTION}

The $6300-\AA$ region of the spectra of comets is important to study yet difficult to interpret. The spectrum in this region is dominated by the emissions from three principle sources: terrestrial airglow $O\left({ }^{1} D\right)$, cometary $O\left({ }^{1} D\right)$, and many members of the $(0,8,0)$ band of cometary $\mathrm{NH}_{2}$. The emission of cometary $O\left({ }^{1} \mathrm{D}\right)$ is important because its source is found in the photodissociation of cometary parent water molecules (Biermann and Trefftz 1964, Festou and Feldman 1981) in the inner coma $\left(<10^{5} \mathrm{~km}\right)$ and of cometary OH (van Dishoeck and Dalgarno 1984, MageeSauer et al. 1989) in the outer coma. Understanding both the absolute abundance and the spatial distribution of $\mathrm{NH}_{2}$ in comets is important because of its likely production from photodissociating parent ammonia molecules. The relative abundance of cometary ammonia is an important indicator of the cosmochemistry associated with the conditions in the early solar nebula where cometary nuclei were formed (Delsemme 1982). The difficulty in studying these emissions arises from the fact that most cometary observations are made with low to medium spectral resolution where all three emissions are blended into a single broad feature.

The terrestrial $O\left({ }^{1} \mathrm{D}\right)$ airglow emission is usually separated from the cometary emissions by recording the spectrum of a region of the nearby sky and subtracting its intensity contribution from the blend. In the case of longslit spectra this can sometimes be accomplished by taking the contribution to the spectrum at the far end of the slit as a measure of the sky (Delsemme and Combi 1979, Fink and Johnson 1984). At low to medium resolution the separation of the $\mathrm{NH}_{2}$ and $\mathrm{O}\left({ }^{1} \mathrm{D}\right)$ is more difficult. Spinrad (1982) and Delsemme and Combi (1983) have shown that the $\mathrm{NH}_{2}$ contributions in the region between the 6300 and the $6364 \AA$ blend (also of $\mathrm{O}\left({ }^{1} \mathrm{D}\right.$ ) and $\mathrm{NH}_{2}$ ) might be scaled to yield an estimate of the $6300 \AA \mathrm{NH}_{2}$ emission.

Sufficiently high spectral resolution can effectively eliminate the problem altogether by enabling all of the various features to be separated spectrally. Arpigny and co-workers have most recently published a set of high resolution coudé and coudé-echelle spectra of the $6300-\AA$ regions of comets Kohoutek (1973 XII), Bradfield (1974 III), Kobayashi-Berger-Milon (1975 IX), West (1976 VI), and Halley (Arpigny et al. 1987). In their work the relative contributions of a number of sets of individual rotational lines of the $\mathrm{NH}_{2}(0,8,0)$ band were compared with one 
another. They found significant variations in the relative strengths of the $\mathrm{NH}_{2}$ emissions in the $(0,8,0)$ band both from comet-to-comet and from day-to-day. This variation contributes an additional uncertainty to the estimate of the $\mathrm{NH}_{2}$ emission at $6300 \AA$ from the measured contribution at $6334 \AA$. They found that the ratio of the $\mathrm{NH}_{2}$ contribution at $6300 \AA$ to that at $6334 \AA$ varied by more than a factor of 3 for different comets on different days. Their spectral resolution corresponded to a spectral element size of approximately $0.15 \AA$. The problem is exacerbated in Halley because of the relatively high abundance of $\mathrm{NH}_{2}$ compared with other comets, but is usually overcome by averaging a numbers of features near 6334 and $6300 \AA$ having mixed upper vib-rotational levels (Fink, private communication).

In this paper we present the results of the analysis of a set of high-resolution spectra of the $6300-\AA$ region of Comet Halley taken with the echelle/reticon system on the 61-inch telescope at the Harvard-Smithsonian Oak Ridge Observatory. These spectra were recorded not only centered on the nucleus but also at various displacements from and around the nucleus. From these observations we have studied the identifications of and the variations of relative intensity between the various members of the $(0,8,0)$ band. The variations were measured not only from day-to-day but also from one location in the coma to another. Results concerning the circular symmetry of the coma as well as limited information regarding the radial brightness profiles of $\mathrm{NH}_{2}$ and $\mathrm{O}\left({ }^{1} \mathrm{D}\right)$ are also presented.

\section{OBSERVATIONS}

The observations of Comet $\mathrm{P} / \mathrm{Halley}$ were taken during the preperihelion period from 23 October 1985 through 6 January 1986 using the linear reticon array with the echelle spectrograph on the 61-inch Wyeth telescope at the Harvard-Smithsonian Oak Ridge Observatory in Harvard, Massachusetts (Latham 1985). At $6300 \AA$ we obtain an approximate spectral resolving power of better than 65,000 or spectral resolution element somewhat less than $0.1 \AA$. The entrance slit to the spectrograph projected a rectangle on the sky with its length running along the north-south line which was 6 by 1.5 arcsec. Table I provides a summary of all the individual 6300 - $\AA$ region scans. As can be seen in the table, spectra were recorded not only with the spectrograph slit centered on the nucleus but also with the slit displaced in different directions and by different amounts. The comet and slit were monitored with the guide TV camera at the focal plane. The slit location with respect to the nucleus was determined by the observer's judgment of the center of brightness of the comet and the known scale of the TV image.

All of the spectra have been "flat-fielded" using a broadband continuum source. Data reduction followed
TABLE I

Oak Ridge Observatory Reticon/Echelle Spectra of the 6300- $\AA$ Region of Comet P/Halley

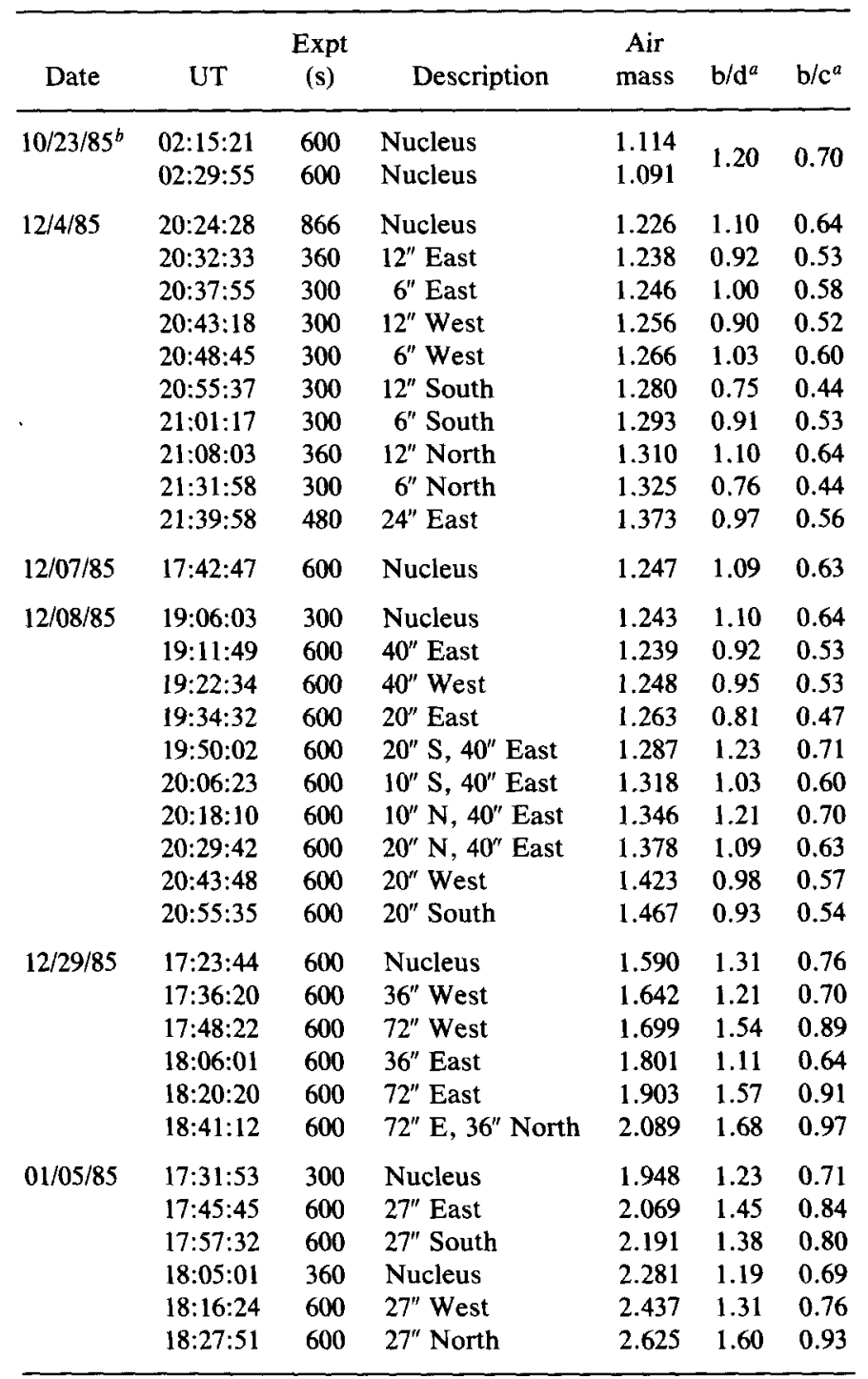

a The results for 23 October were summed before ratios were calculated.

${ }^{b}$ Ratio of $\mathrm{NH}_{2} \mathrm{~b} / \mathrm{d}$ and $\mathrm{b} / \mathrm{c}$ features. (See text for details.)

the procedures described by Wyatt (1985). Although no formal absolute calibrations were made for the spectra, relative fluxes can and have been derived between spectra taken on the same night. Standard air mass corrections have been used for this purpose, and the consistency of the results between spectra is taken as a validity of our approach. Typically the air mass did not change much between spectra and the differences between corrections from spectrum to spectrum on one night were small. See Table II for information about observational geometry. Integrated line fluxes were calculated for various fea- 


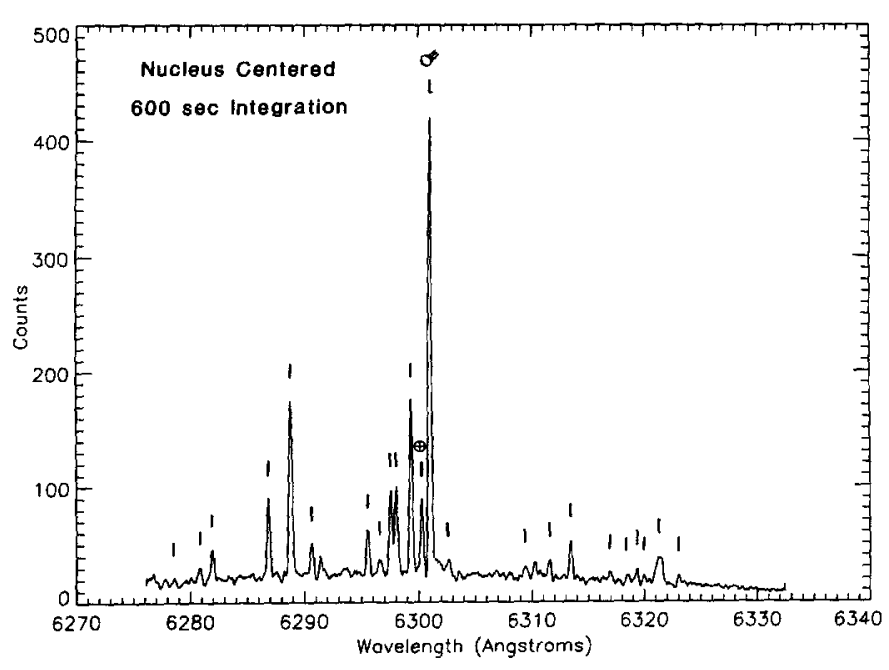

FIG. 1. Echelle-Reticon scan of the $6300-\AA$ region of the spectrum of Comet P/Halley. The many features of the $(0,8,0)$ band of $\mathrm{NH}_{2}$ are marked with a short vertical line and the $O\left({ }^{\prime} D\right)$ lines from airglow and the comet are indicated with $\mathrm{a} \oplus$ and a $O \equiv$, respectively.

tures and groups of features as will be presented later in this paper. The spectral resolving power is so high that the contribution to each spectral pixel from the dust-scattered solar continuum is very small as compared with any of the emission lines. This is even the case in the nucleuscentered spectra where the relative continuum contribution is the largest. The choice of the appropriate continuum level is not at all critical as it amounts to no more than a few percent of the line flux even for the strong lines or groups of lines for which relative line fluxes are presented in this paper. A smooth continuum was estimated from a few regions spaced across the spectrum which were free of emission line contributions. Changing the procedure had little effect on the relative line fluxes anyway.

Figure 1 shows an example of one of the nucleus-centered scans taken on 29 December 1985 . The $O\left({ }^{1} D\right)$ lines from both the airglow and the comet are indicated, as well as are the positions of numerous members of the $\mathrm{NH}_{2}$ $(0,8,0)$ band. The counts scale on the ordinate represents an arbitrary flux scale and not true counts in a statistical sense because of the air mass correction and the flatfielding procedure employed.

\section{RESULTS}

\section{Identification of Spectral Features in the 6300-A Region}

Twenty-one members or blends of members of the $\mathrm{NH}_{2}$ $(0,8,0)$ band have been identified using the laboratory wavelengths and identifications of Dressler and Ramsey (1959) and Woszczyk (1962). All of the features' wave-
TABLE II

Observational Geometry for the Oak Ridge Observatory Echelle/Reticon Spectra

\begin{tabular}{lccccc}
\hline \multicolumn{1}{c}{ Date } & $r(\mathrm{AU})$ & $\dot{r}(\mathrm{~km} / \mathrm{sec})$ & $\Delta(\mathrm{AU})$ & $\dot{\Delta}(\mathrm{km} / \mathrm{sec})$ & $\beta\left({ }^{\circ}\right)$ \\
\hline $10 / 23 / 85$ & 2.05 & -23.94 & 1.34 & -53.19 & 24.4 \\
$12 / 4 / 85$ & 1.44 & -26.25 & 0.66 & +17.40 & 36.0 \\
$12 / 7 / 85$ & 1.39 & -26.39 & 0.69 & +23.14 & 41.3 \\
$12 / 8 / 85$ & 1.36 & -26.48 & 0.72 & +26.22 & 44.3 \\
$12 / 29 / 85$ & 1.04 & -26.62 & 1.12 & +34.31 & 54.1 \\
$1 / 5 / 86$ & 0.93 & -25.96 & 1.25 & +31.55 & 50.9 \\
\hline
\end{tabular}

Note. $r$, heliocentric distance in $\mathrm{AU} ; \dot{r}$, heliocentric velocity in $\mathrm{km} / \mathrm{sec}$; $\Delta$, geocentric distance in $A U ; \dot{\Delta}$, geocentric velocity in $\mathrm{km} / \mathrm{sec} ; \beta$, Earth-comet-Sun angle in degrees.

lengths and laboratory identifications are listed in Table III along with the comet and airglow $O\left({ }^{1} D\right)$ lines. In several cases Woszczyk provided the wavelengths of a line attributed to $\mathrm{NH}_{2}$ but no transition identification is given. These are denoted in the table with the entry W62 in the identification column.

Because these spectra are of such a high resolution, note that two moderately strong lines, which have been associated by observers (e.g., Magee-Sauer et al. 1989) with the ${ }^{\mathrm{R}} Q_{0, \mathrm{~N}}$ branch from lower resolution spectra are actually found to be members of the ${ }^{\mathrm{P}} R_{2, \mathrm{~N}-2}$ branch. These are the cometary features at wavelengths of 6294.86 and $6295.90 \AA$. In addition to those features listed, there is evidence of the presence of some very weak lines very near the cometary $O\left({ }^{1} D\right)$ lines which are members of the ${ }^{R} Q_{0, N}$ branch.

\section{Relative Fluxes within the $\mathrm{NH}_{2}(0,8,0)$ Band}

Arpigny et al. (1987) compared the fluxes of several groups of $\mathrm{NH}_{2}$ lines with each other. Of particular note was the comparison of the $\mathrm{NH}_{2}$ contributions to the emission in the immediate vicinity of $6300 \AA$ with that in the vicinity of $6334 \AA$. As mentioned earlier, the flux at 6334 $\AA$ has been used by low to medium resolution observers to assess the flux contribution of $\mathrm{NH}_{2}$ at $6300 \AA$. The reticon/echelle scan centered on $6300 \AA$ does not extend quite far enough to the red to cover the strong $\mathrm{NH}_{2}$ lines at 6333 and $6336 \AA$ in question which Arpigny et al. designated as feature " $c$ " in their notation. However, the groups of features between 6285 and $6293 \AA$ and 6295 and $6302 \AA$, which they designate as features " $d$ " and "b," respectively, as well as the oxygen line, designated as " $a$ " are all present in our spectra. Arpigny (private communication) reports that the " $d$ " features, which appear both in our spectra and theirs, and the " $c$ " features at $6334 \AA$ which appear only in theirs originate from the same upper rotational levels. In fact their results for the $\mathrm{d} / \mathrm{c}$ ratio, not published but reported to us privately, show 
that its value was found to be constant in their data and is given by them to be $0.58 \pm 0.04$. Half, or 0.02 , of the 0.04 uncertainty represents the possibility of a systematic error in the ratio, whereas only the remaining 0.02 represents the $3-\sigma$ deviation in the mean for all measured values. In other words the data show that the value is in fact constant to within better than $4 \%$ but there is an additional $4 \%$ uncertainty in what that constant value is. It is also important to note that this average was constant for the eight different comets where the heliocentric distances varied from 0.58 to $2.08 \mathrm{AU}$ and the heliocentric velocities varied from -30 to $+45 \mathrm{~km} \mathrm{sec}^{-1}$, but in the same data sets the $b / c$ (and $b / d$ ) ratio varied by over a factor of 3 . Therefore, with confidence we can use our results for $d / b$ and directly have a measure of $b / c$.

Table I shows our values of the b/d ratio and the calculated b/c ratio (using Arpigny's value of $0.58 \mathrm{for} d / c$ ). It is apparent that while our values for the $b / d$ and $b / c$ in the nucleus-centered spectra do vary over time like the results reported by Arpigny et al., they do not show as large a range of variation. However, our data do not span as much of a range of heliocentric distance, heliocentric velocity, or gas production rate as do theirs. It is encouraging that for the cluster of observations taken in early December, i.e., on the 4 th, 7 th, and 8 th, that the ratios for the nucleus-centered observations are nearly identical $(0.64$, 0.63 , and 0.64 , respectively). This does provide encouraging support for the quality of the data and the reduction procedures used. For this reason we believe that the $20 \%$ range in the variation of the ratio is real and not just statistical scatter. Finally, although we have no coincident or nearly coincident observations with those of Arpigny et al. (1987), our $\mathrm{d} / \mathrm{b}$ ratios (and computed $\mathrm{b} / \mathrm{c}$ ratios) fall well within the much larger range they found for several comets. Therefore, their is no overwhelming reason to be suspicious of these results.

We find that in addition to the small variation of the $\mathrm{b} / \mathrm{c}$ ratio over time there is even more variation in the ratio at different locations within the coma. Because of the lower signal-to-noise in the displaced spectra (non-nucleus-centered) there is considerable scatter in the ratios although the existence of the variation is unmistakable. In order to help this situation the data corresponding to spectra taken at the same displacement from the nucleus but in different directions were summed and then new average ratios were calculated. These average ratios are plotted as a function of distance from the nucleus (in kilometers) in Fig. 2. It appears that the ratio of $b / c$ (again inferred from $b / d$ ) increases with increasing distance from the nucleus.

Different upper rotational levels are responsible for the $b$ features than for the $d$ and $c$ features. Differences in the populations of different upper rotational levels could be caused by two factors. One possibility is a SwingsGreenstein effect in which the solar fluorescence mecha-

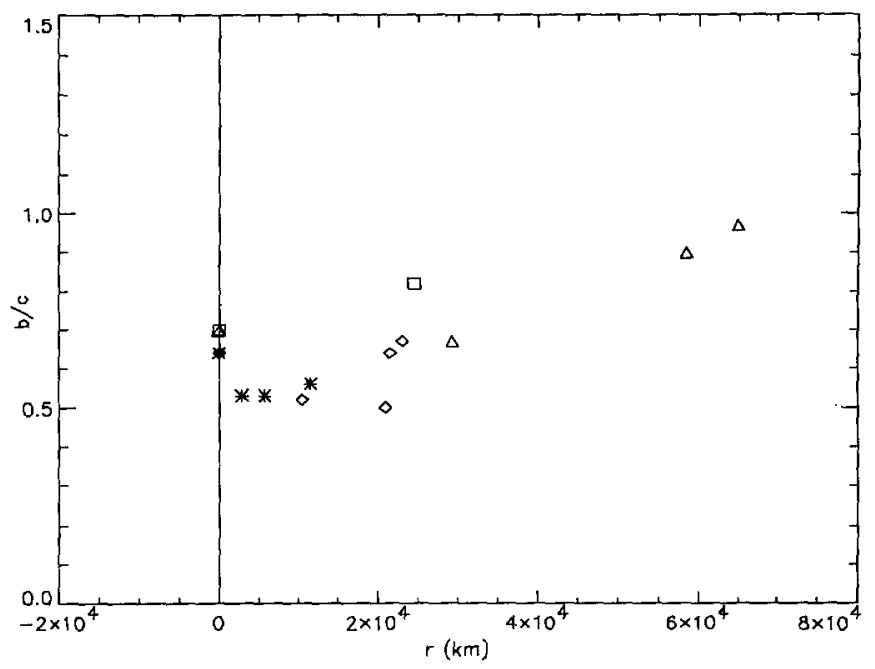

FIG. 2. The radial dependence of the $b / c$ ratio on Comet Halley. The ratio of $b / c$ as a function of distance from the nucleus is plotted. The symbols used are as follows: asterisk for December 4, diamond for December 8, triangle for December 29, and square for January 5 . It appears that the ratio first decreases from 0.65 at the nucleus to 0.52 at 5000 to $10,000 \mathrm{~km}$ and then rises gradually to a value of 1.0 at 60,000 $\mathrm{km}$.

nism responsible for the emission of $\mathrm{NH}_{2}$ is dependent upon the heliocentric velocity at different locations within the coma owing to the presence of absorption lines in the solar spectrum. This has been shown to be important for OH (Schleicher 1983) and CN (Malaise 1970), for example. A second more likely possibility, at least for the case of $\mathrm{NH}_{2}$, is variation in the rotational levels due to variations in rotational temperature with radial distance in the coma.

Even though no detailed fluorescence equilibrium calculation has ever been done for $\mathrm{NH}_{2}$ we favor the latter explanation for two reasons. First, the variation is dependent on distance from the nucleus rather than on sunward-antisunward displacement. The typical SwingsGreenstein effect normally shows itself as a sunward to antisunward asymmetry in the relative values of certain sensitive rotational lines. Quite obviously this is caused by the fact that along any particular line of sight the sunward half of the coma is moving sunward and the antisunward half is moving antisunward, each with a dispersion around some projected fraction of the average outflow speed. Second, there are no sets of strong absorption lines in the region of the solar spectrum responsible for the $\mathrm{NH}_{2}$ fluorescence as there are for the cases of both $\mathrm{CN}$ and $\mathrm{OH}$.

Calculated spectra of the rotational structure of the $\mathrm{NH}_{2}$ $(0,8,0)$ band do in fact show temperature dependence. Figure 3 shows a set of simple spectra from Wyckoff (private communication) corresponding to the range of 

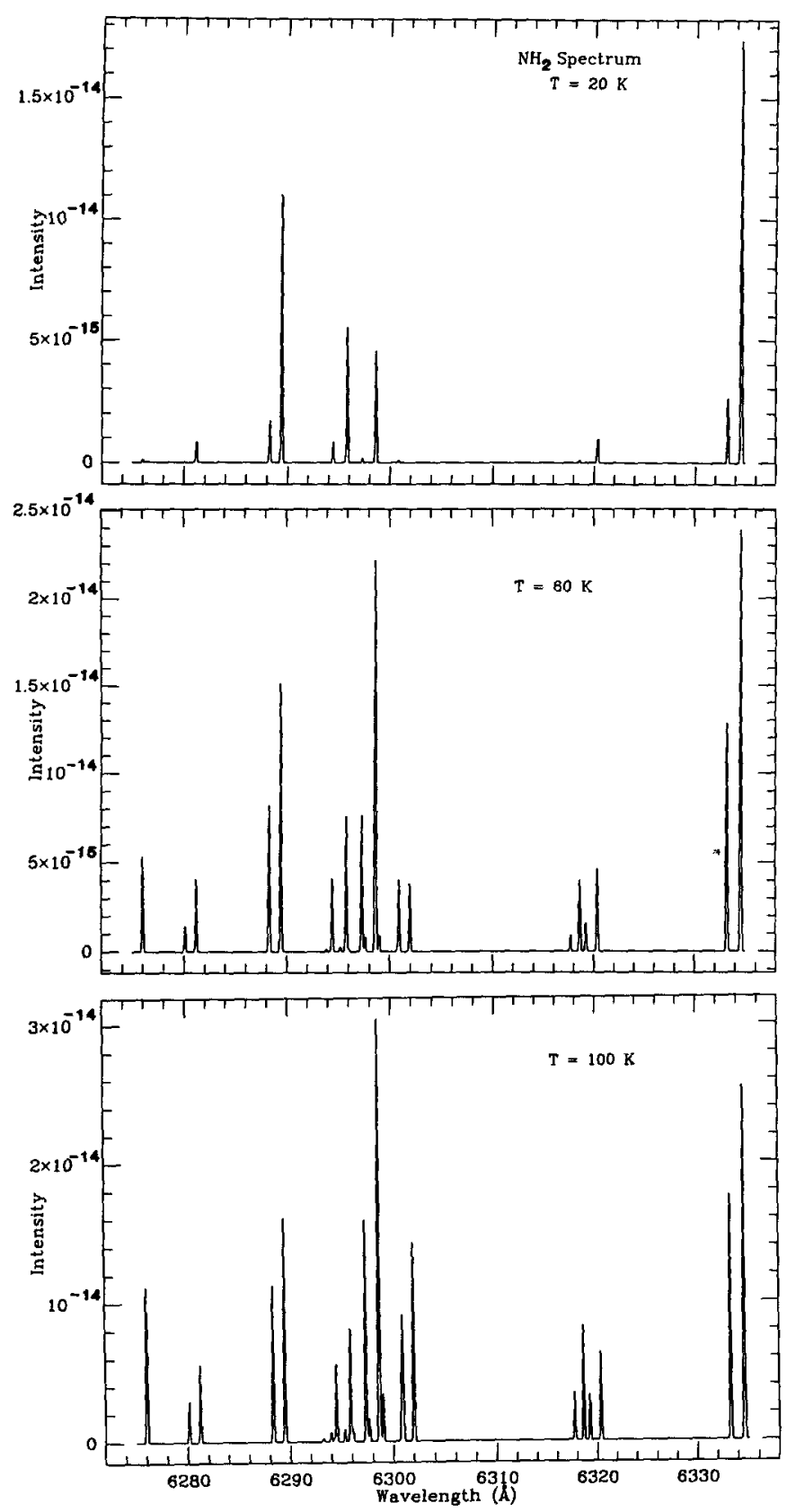

FIG. 3. Calculated fluorescence spectra of the $\mathrm{NH}_{2}(0,8,0)$ band. The simple fluorescence spectrum of the $\mathrm{NH}_{2}(0,8,0)$ band calculated by Wyckoff (private communication) for rotational temperatures of 20, 60, and $200 \mathrm{~K}$. The spectrum at $60 \mathrm{~K}$ gives the best overall match to the observed spectra.

temperatures one might expect to occur in the coma. The calculation is a very simple calculation since it assumes only a flat continuum excitation source, rather than a true solar spectrum. All of the observed spectra seem to agree best with a temperature near 50-60 K. Wyckoff (private communication) reported that most of the spectra pub- lished by Arpigny et al. (1987) were best matched by the calculated spectrum at $35 \mathrm{~K}$. The differences we find between various observed spectra are still smaller than those between the observed and these simple synthetic spectra so it is not yet possible to do any kind of detailed spectral model analysis. There are important points to be made. The rotational temperatures implied are definitely of the magnitude both found in the coma observationally (Bockelée-Morvan and Crovisier 1987; Weaver et al. 1987) and expected theoretically (Combi 1989). There are measureable differences in the rotational structure which are indicative of and consistent with differences either in rotational temperature or in the relative outflow speed in different parts of the coma. For a number of reasons we favor the variation in rotational temperature. Therefore, careful modeling of observed spectra of the $\mathrm{NH}_{2}$ band structure as a function of distance from the nucleus could provide an important diagnostic tool for the remote sensing of temperatures in the comae of comets.

\section{Circular Symmetry of the Coma}

In addition to the relative contributions of $\mathrm{NH}_{2}$ and $\mathrm{O}\left({ }^{1} \mathrm{D}\right)$ features, these data also provide information regarding the circular symmetry of the coma as well as some limited information of the radial brightness distribution. The offset spectra taken at the same distance from the nucleus but in different directions serve as an indication of the circularity of the isophotes which says something about the sphericity of the coma, or at least of the $\mathrm{NH}_{2}$ and $O\left({ }^{1} D\right)$ in the coma. For the December and January data, where the offset observations were made, the sunward direction was West to West-Southwest of the nucleus. The important and unique value in these data is that the oxygen observations are clearly spectrally uncontaminated. In nearly all other types of spatially resolved observations (medium resolution long-slit spectra and narrowband $\mathrm{CCD}$ images), the $\mathrm{NH}_{2}$ and $\mathrm{O}\left({ }^{1} \mathrm{D}\right)$ contributions have to be separated using the Spinrad (1982) method which the results of Arpigny et al. (1987) and those presented here indicate may not always be reliable.

The importance of the unambiguous observations of $O\left({ }^{1} D\right)$ especially cannot be understated. It is now well accepted that the emission of these photons $(6300$ and $6364 \AA$ ) results from a forbidden transition of oxygen atoms produced in the ${ }^{1} \mathrm{D}$ state upon photodissociation of the parent molecule. The atom then undergoes a spinforbidden decay to the ground ${ }^{3} \mathrm{P}$ state after a lifetime of roughly $100 \mathrm{sec}$. The emission of these photons thus provides a direct trace of the distribution of the parent molecule. In the inner coma $\left(<10^{5} \mathrm{~km}\right)$ the principal source of these atoms is the photodissociation of water. In the outer coma the principal source is the photodissociation of OH (van Dishoeck and Dalgarno 1984, Magee- 
Sauer et al. 1989). The spatial region covered by our observations is clearly the inner water source. A further complication in the very innermost coma is that the ${ }^{1} \mathrm{D}$ state can be collisionally quenched before the photon emission. This however is generally too close to the nucleus to be observable for comet-Earth distances which are at least a few tenths of an AU (Festou and Feldman 1981).

Table IV lists the integrated line fluxes in arbitrary units of the $\mathrm{O}\left({ }^{1} \mathrm{D}\right)$ and $\mathrm{NH}_{2}$ emissions in all of the offset spectra. The $\mathrm{NH}_{2}$ values are the sums of the $b$ and $d$ features. The main apparent departure from circularity, which can be seen by a quick examination of the table, is that the north and south displacements are generally larger than the east and west values. The differences are particularly noticeable for the smallest displacements. This effect however is only due to the fact that when a rectangular slit is oriented with its longer dimension along the north-south direction, it samples distances both closer to and farther from the nucleus than the displacement of the center, whereas when the same slit is displaced to the east or west it samples distances only greater than or equal to the nominal center position. Therefore corresponding points to the north and south are always brighter than those to the east and west. For the $O\left({ }^{1} D\right)$ emission which approximately follows a $1 / r$ dependence, this slit function can be simply integrated and accounts very well for all of the differences between north-south and east-west.

The more important asymmetry would be a sunward to anti-sunward one which would be indicative of any preferential sunward ejection of material which has been reported by a number of investigators. The most dramatic asymmetries were seen in the dust distribution in the Giotto Multicolor camera (Keller et al. 1987), however it is well understood both observationally and theoretically that the dust collisionally decouples from the gas in the inner coma (Combi 1989).

Asymmetries have been reported both in the spatial and velocity distributions of coma gases. Net sunward doppler shifts of up to a $0.3 \mathrm{~km} \mathrm{sec}^{-1}$ have been seen in radio and infrared observations of parent and daughter species in the coma (Larson et al. 1986, Schloerb et al. 1987; TacconiGarman 1989). Various instruments on the VEGA spacecraft also reported asymmetric distributions in gas density through comparison of inbound and outbound data, for example, with TKS by Krasnopolvsky et al. (1987), and with NGE by Hsieh et al. (1987).

In the data presented here similar asymmetries would show themselves as east/west brightness differences. An examination of the west to east brightness ratio from the $\mathrm{NH}_{2}$ and $\mathrm{O}\left({ }^{\prime} \mathrm{D}\right)$ observations yields some interesting results. We find an average for the sunward to anti-sunward ratio of 1.04 for $O\left({ }^{1} D\right)$, which represents the water distribution, and 0.99 for $\mathrm{NH}_{2}$. The values show corresponding
TABLE III

Spectral Features in the $6300-\AA \AA$ Region

\begin{tabular}{|c|c|c|c|}
\hline$\lambda_{\text {air }}(\text { comet })^{a}$ & $\lambda_{\text {air }}(\mathrm{lab})^{b}$ & \multicolumn{2}{|c|}{ Identification $^{c}$} \\
\hline 6277.89 & $\begin{array}{l}6277.88 \\
6278.14\end{array}$ & ${ }^{\mathrm{P}} P_{1, \mathrm{~N}-\mathrm{I}}$ & $4_{04}-5_{14}(0,11,0)$ \\
\hline $\begin{array}{l}6280.09 \\
6281.20\end{array}$ & $\begin{array}{l}6280.11 \\
6281.20\end{array}$ & ${ }^{\mathrm{R}} R_{\mathbf{0 , \mathrm { N }}}$ & $2_{11}-1_{01}$ \\
\hline 6286.13 & 6286.14 & ${ }^{\mathrm{R}} \boldsymbol{R}_{\mathbf{0}, \mathrm{N}}$ & $1_{10}-0_{00}$ \\
\hline 6288.06 & 6288.05 & W62 ${ }^{d}$ & \\
\hline 6289.93 & 6289.97 & W62 & \\
\hline 6294.86 & $\begin{array}{l}6294.45 \\
6294.82\end{array}$ & ${ }^{\mathrm{P}} \boldsymbol{R}_{2, \mathrm{~N}-2}$ & $4_{13}-3_{21}$ \\
\hline 6295.90 & $\begin{array}{l}6295.83 \\
6296.08\end{array}$ & $\begin{array}{l}\text { W62 } \\
{ }^{\mathrm{P}} \boldsymbol{R}_{2, \mathrm{~N}-2}\end{array}$ & $3_{12}-2_{20}$ \\
\hline 6296.89 & 6296.85 & W62 & \\
\hline 6297.31 & 6297.32 & ${ }^{\mathrm{R}} Q_{0, \mathrm{~N}}$ & $2_{12}-2_{02}$ \\
\hline 6298.60 & 6298.62 & ${ }^{\mathrm{R}} Q_{0, \mathrm{~N}}$ & $2_{12}-2_{03}$ \\
\hline 6300.30 & 6300.30 & $O\left({ }^{1} D\right)$ & \\
\hline 6300.28 & 6300.30 & $O\left({ }^{1} D\right)$ & \\
\hline 6301.91 & $\begin{array}{l}6301.86 \\
6301.97\end{array}$ & W62 & \\
\hline 6308.70 & 6308.71 & W62 & \\
\hline 6310.80 & 6310.78 & W62 & \\
\hline 6312.74 & 6312.71 & W62 & \\
\hline 6316.22 & 6316.06 & W62 & \\
\hline 6317.81 & 6317.72 & ${ }^{\mathrm{P}} Q_{2, \mathrm{~N}-1}$ & $2_{11}-2_{21}$ \\
\hline 6318.63 & 6318.63 & ${ }^{\mathrm{P}} Q_{2, \mathrm{~N}-1}$ & $2_{11}-2_{21}$ \\
\hline 6319.26 & 6319.25 & ${ }^{\mathrm{P}} Q_{2, \mathrm{~N}-2}$ & $22_{12}-2_{20}$ \\
\hline 6320.59 & $\begin{array}{l}6320.59 \\
6320.76\end{array}$ & ${ }^{\mathrm{P}} Q_{2, \mathrm{~N}-1}$ & $3_{12}-3_{22}$ \\
\hline 6322.34 & 6322.33 & ${ }^{\mathrm{P}} Q_{2, \mathrm{~N}-\mathrm{l}}$ & $3_{12}-3_{22}$ \\
\hline
\end{tabular}

${ }^{a}$ Rest wavelength in air of cometary spectrum feature in Angstrom units.

${ }^{b}$ Laboratory wavelength in air in Angstrom units.

c Line identifications for $\mathrm{NH}_{2}$ are from Dressler and Ramsey (1959) and/or Woszczyk (1962). Except for the feature at 6277.89, all $\mathrm{NH}_{2}$ lines are members of the $(0,8,0)$ band.

${ }^{d}$ W62 signifies lines attributed to $\mathrm{NH}_{2}$ by Woszczyk (1962) with no specified transition designation.

${ }^{e}$ Rest wavelengths in air of $O\left({ }^{l} D\right)$ line.

statistical deviations taking all of the ratios as a group (to average out the point to point scatter) of 0.04 and 0.06 . These are $3 \sigma$ in the means of the distributions. It is worth noting that even though for $O\left({ }^{1} D\right)$ where the asymmetry is of the order of the uncertainty that in every individual case the west to east ratio is always greater than one. Therefore we feel there is a small consistent sunward bias 
to the water distribution. The fact that the $\mathrm{NH}_{2}$ distribution is not asymmetric may be due to the fact that it is a daughter species. Even if its parent (presumably $\mathrm{NH}_{3}$ ) were $4 \%$ asymmetric, the vectorial ejection of the $\mathrm{NH}_{2}$ should generally isotropicize the distribution.

The fact that we do not see some of the dramatic asymmetries reported in the coma of Halley's comet is not surprising if we are careful to compare only with observations made during the preperihelion period. The $\mathrm{OH}$ radio observations are the best long-term observations with which to compare since they cover both the pre- and postperihelion periods. Tacconi-Garman (1989) has analyzed the observed $\mathrm{OH}$ doppler line profiles determined from $18-\mathrm{cm}$ radio observations using a model parameterized with an anisotropy parameter (AP). The parameter has a value of zero if the coma is spherical and a positive value if the coma is skewed sunward. Whereas he finds substantial sunward offsets for observations of Halley, especially in the period of March and April 1986 when most of the other observations were taken, his December 1985 and January 1986 values are $(0.25 \pm 0.18)$ and $(0.02 \pm 0.12)$. Therefore, we should expect to find a nearly spherical distribution throughout the period covered by our observations. It is also noteworthy to mention here that Tacconi-Garman also found significant asymmetries in Halley and other comets at certain times with values for AP greater than unity; but few negative values were found.

\section{Radial Brightness Distributions of $\mathrm{O}\left({ }^{1} \mathrm{D}\right)$ and $\mathrm{NH}_{2}$}

Since the individual observations in different directions show at most only a small departure from circularity, we have constructed average radial brightness distributions for $\mathrm{O}\left({ }^{1} \mathrm{D}\right)$ and $\mathrm{NH}_{2}$ by averaging over different directions. The radial profiles are shown in Figs. $4 \mathrm{a}-4 \mathrm{c}$. The $\mathrm{NH}_{2}$ profiles were constructed again from the sums of $b$ and $d$ features. The horizontal error bars were derived from the sums of the range of distances covered by the slit, and an estimate of the uncertainty in pointing accuracy relative to the location of the photonucleus. For the data from December 4 and 8 there were enough widely separated points to attempt a crude model analysis for both species. For the December 29 data there were only enough points to verify the approximate $1 / r$ distribution for $O\left({ }^{1} D\right)$ expected.

The $O\left({ }^{1} \mathrm{D}\right)$ profiles for December 4 , and 8 , and 29 are consistent with the expected $1 / r$ distribution. During early December the comet's heliocentric distance was about 1.4 AU, which would place the water photodissociation scale length at about $1.6 \times 10^{5} \mathrm{~km}$. Given the limited spatial extent of the observed profile it is impossible to distinguish a profile with this lifetime from one from an infinite lifetime.
TABLE IV

Circular Symmetry of the Coma of Comet Halley

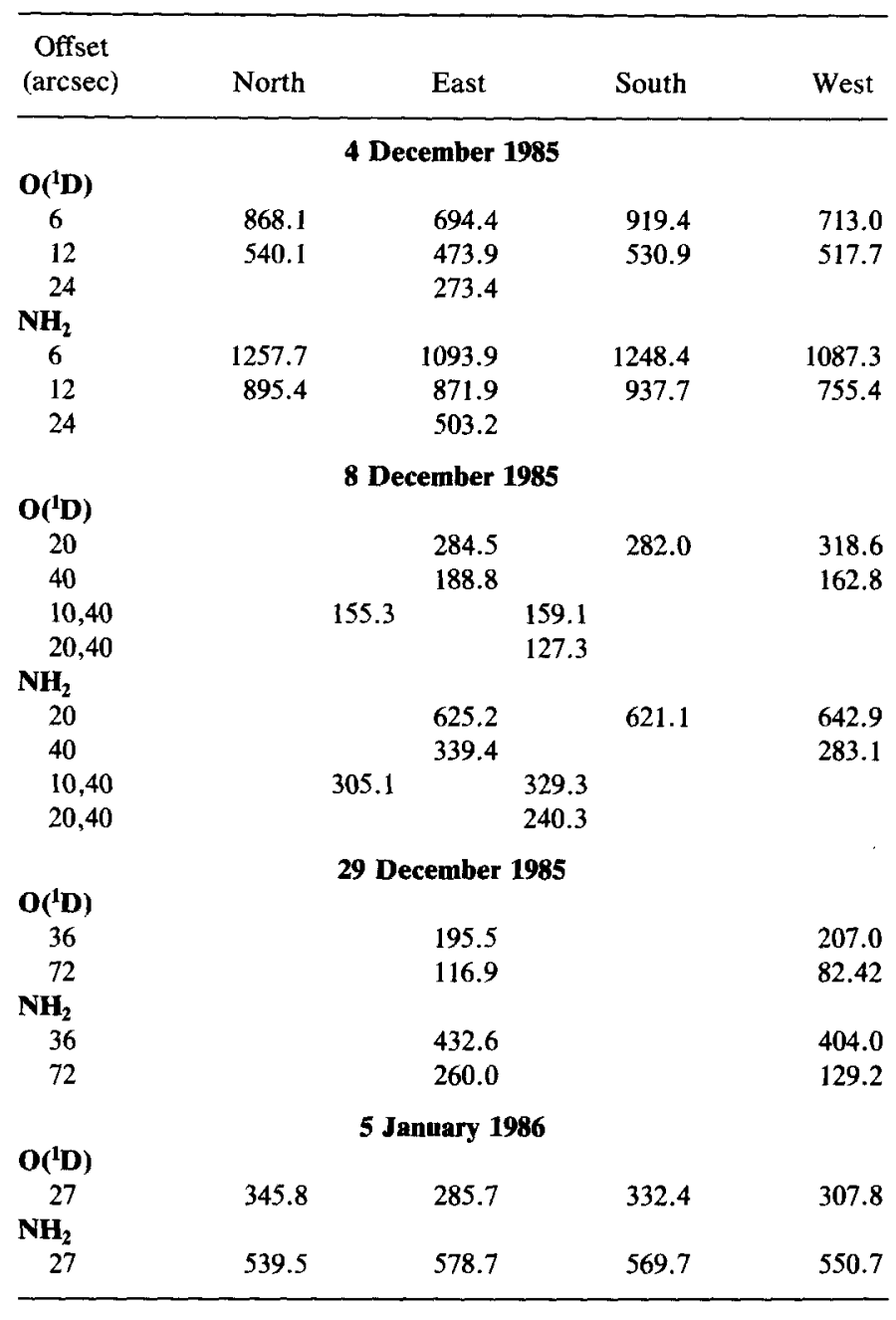

In the few $\mathrm{NH}_{2}$ brightness profiles which have been published in the literature and analyzed, the parent and daughter scale lengths were found to be nearly the same (Delsemme and Combi 1983; Johnson et al. 1984; Konno and Wyckoff 1988), or to differ by as much as a factor of $50 \%$ (Wyckoff et al. 1988). Given this fact it is possible to force-fit a Haser model in which both scale lengths are the same, even for our sparse profiles for December 4 and 8. The best fit equal-scale-length Haser models are shown as the dashed curved lines in Figs. $4 \mathrm{a}$ and $4 \mathrm{~b}$. The scale lengths determined for those two profiles are $(3.7 \pm$ $1.3) \times 10^{4}$ and $(2.6 \pm 0.7) \times 10^{4} \mathrm{~km}$, respectively. When reduced to $1 \mathrm{AU}$ (assuming they vary as the square of the heliocentric distance) they are consistent with the average of the parent and daughter values at $1 \mathrm{AU}$ of 1.1 and $1.6 \times$ $10^{4}$ for Comet Halley (Wyckoff et al. 1988), the equal parent and daughter values reported by Delsemme and Combi (1983) for Comet Kohoutek at $0.465 \mathrm{AU}$ of $6.9 \times$ 

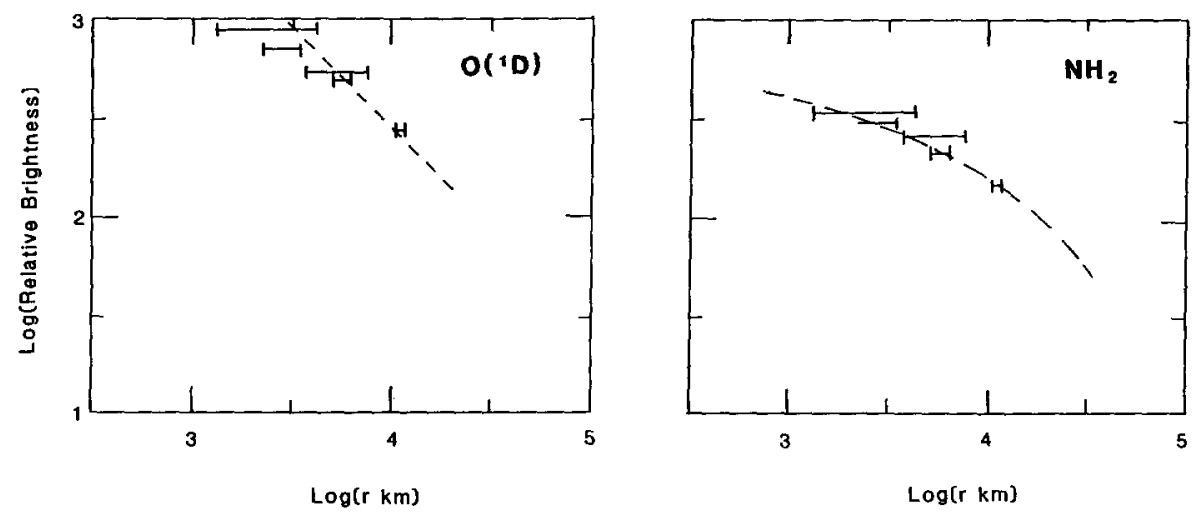

FIG. 4a. $\mathrm{O}\left({ }^{1} \mathrm{D}\right)$ and $\mathrm{NH}_{2}$ radial brightness profiles in Comet Halley on 4 December 1985 . The brightness values are plotted as error bars whose radial range corresponds to the sum of the slit coverage and the slit location uncertainty. The dashed line on the $O\left({ }^{1} \mathrm{D}\right)$ profile corresponds to a best-fit $1 / r$ profile which is expected for the distance range covered. The dashed line on the $\mathrm{NH}_{2}$ profile corresponds to a best-fit equal-scale-length Haser model where the scale length $=(3.7 \pm 1.3) \times 10^{4} \mathrm{~km}$.
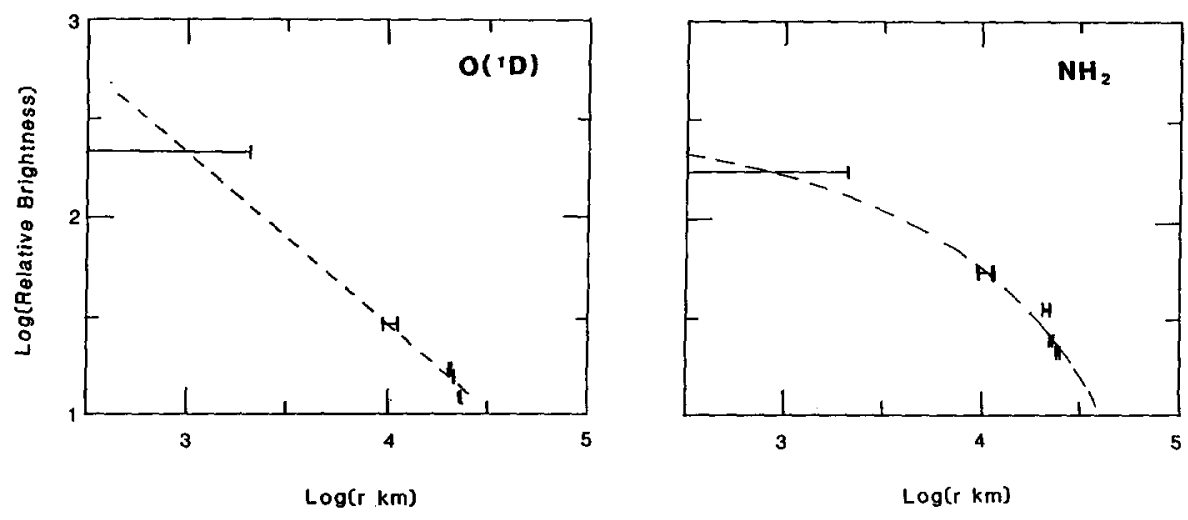

FIG. 4b. $\mathrm{O}\left({ }^{1} \mathrm{D}\right)$ and $\mathrm{NH}_{2}$ radial brightness profiles in Comet Halley on 8 December 1985. The best-fit equal-scale-length Haser model for $\mathrm{NH}_{2}$ corresponds to a scale length of $(2.6 \pm 0.7) \times 10^{4} \mathrm{~km}$. Refer to Fig. 4a for other details.
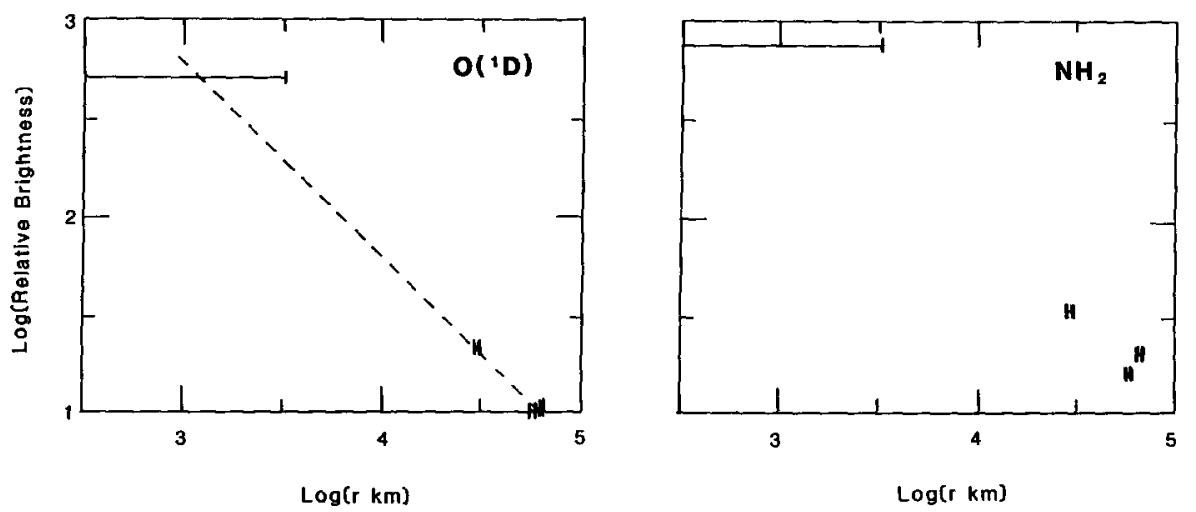

FIG. 4c. $\mathrm{O}\left({ }^{\mathrm{I}} \mathrm{D}\right)$ and $\mathrm{NH}_{2}$ radial brightness profiles in Comet Halley on 29 December 1985. No equal-scale-length Haser model for $\mathrm{NH}_{2}$ was possible. Refer to Fig. 4 a for other details. 
$10^{3} \mathrm{~km}$ and the Monte Carlo velocities and lifetimes found by Konno and Wyckoff (1988). It should be stressed that our profiles do not constrain the parent and daughter scale lengths to be equal.

\section{SUMMARY AND CONCLUSIONS}

We have presented the results of the analysis of a set of high-resolution echelle spectra of the 6300 -Å region of Comet $\mathrm{P} /$ Halley taken during the preperihelion period.

1. The identifications of numerous members of the $\mathrm{NH}_{2}$ $(0,8,0)$ band were found and some discrepancies in the literature corrected.

2. The relative intensity of sets of features within the $(0,8,0)$ band have been shown to vary over time, as previously reported by Arpigny et al. (1987), although our results for a more limited set of data showed a smaller range variation than they reported. In late October 1985 and from early December 1985 to early January 1986 the ratio of the $6300-\AA$ contribution of $\mathrm{NH}_{2}$ to that at $6334 \AA$ varied between 0.63 and 0.70 .

3. A new result, which shows that the rotational structure within the $(0,8,0)$ band changes with radial distance from the nucleus within the coma, has also been found. Therefore, a more sophisticated spectral analysis (than attempted here) in combination with similar or better high resolution spectra could yield a new method for remote sensing of temperatures within the coma. This variation of rotational structure also could introduce further uncertainty to the process of separation of the spatial distributions of $\mathrm{O}\left({ }^{1} \mathrm{D}\right)$ and $\mathrm{NH}_{2}$ in low to medium resolution spectra. The $\mathrm{NH}_{2} 6300$ to $6334 \AA$ ratio varied from an average of 0.66 near the nucleus up to 0.90 at a few tens of thousands of kilometers.

4. The $O\left({ }^{1} \mathrm{D}\right)$ distribution, which is a tracer of the primary water distribution shows a small sunward bias $(0-10 \%)$ from spherical symmetry. The $\mathrm{NH}_{2}$ distribution showed no sunward bias; it should however become isotropicized somewhat by the vectorial ejection of the radicals upon their production by the photodissociation of the parent.

5. The $O\left({ }^{1} \mathrm{D}\right)$ distribution is consistent with the $1 / r$ water distribution expected in the inner coma. Simple equalscale-length Haser models for the radial profiles of the $\mathrm{NH}_{2}$ yield values in the range found by other observers for separate parent and daughter values.

The ability to observe the $6300 \AA$ line from ground-based telescopes provides an important monitor of the abundance and spatial distribution of water in comets. The very high spectral resolution results presented here (and by others) show that the separation of the $\mathrm{NH}_{2}$ and $O\left({ }^{1} D\right)$ contributions can be done to some degree of uncertainty. The ratio of the $\mathrm{NH}_{2}$ contribution at $6300 \AA$ and to that at $6334 \AA$ in fact can vary somewhat from day to day, comet to comet, and from location to location within the coma. Caution should be taken when estimating uncertainties both in $\mathrm{O}\left({ }^{\mathrm{l}} \mathrm{D}\right)$ production rates from nucleuscentered observations and in spatial profiles of $O\left({ }^{\prime} D\right)$. Whenever possible high spectral resolution observations should be used as a check on the scaling of one $\mathrm{NH}_{2}$ feature to another.

\section{ACKNOWLEDGMENTS}

We thank GTE Research Laboratories for instrumentation support to the Oak Ridge Observatory. Gunther Schwartz is thanked for providing much more than observing assistance. Support for MRC under Contracts NASW-4170 and NASW-4331 from the Planetary Astronomy Program at NASA is gratefully acknowledged. Much of the work by MRC was performed while at Atmospheric and Environmental Research, Inc. Finally, we thank Drs. C. Arpigny and S. Wyckoff for very helpful discussions and correspondence, and for providing us unpublished information, and Dr. H. Spinrad and an anonymous referee for constructive criticism.

\section{REFERENCES}

Arpigny, C., J. Manfroid, P. Magain, and R. Haefner 1987. On the forbidden red lines of oxygen in comets. In Proceedings of the Symposium on the Diversity and Similarity of Comets, ESA SP 278, 571-576.

Biermann, L., and E. Treffiz 1964. Uber die Mechanismen der Ionisation und der Anregung in Kometen Atmospheren. Z. Astrophys. 59, 1-28.

Bockelee-Morvan, D., ANd J. Crovisier 1987. The $2.7 \mathrm{~mm}$ water band of comet P/Halley: Interpretation of observations by an excitation model. Astron. Astrophys. 187, 425-430.

Comвi, M. R. 1989. The outflow speed of the coma of Halley's comet. Icarus 81, 41-50.

Delsemme, A. H. 1982. Chemical composition of cometary nuclei. In Comets (L. L. Wilkening, Ed.), pp. 85-130. Univ. of Arizona Press, Tucson.

Delsemme, A. H., AND M. R. Combi 1979 . O(' $\left.{ }^{(} D\right)$ and $\mathrm{H}_{2} \mathrm{O}^{+}$in comet Bennett 1970II. Astrophys. J. 228, 330-337.

Delsemme, A. H., AND M. R. Combi 1983. Neutral cometary atmospheres. IV. Brightness profiles in the inner coma of comet Kohoutek (1973 XII). Astrophys. J. 271, 388-397.

Dressler, K., AND D. A. Ramsey 1959. The electronic absorption spectra of $\mathrm{NH}_{2}$ and $\mathrm{ND}_{2}$. Phil. Trans. Soc. London A 251, 553-602.

Festou, M., AND P. D. Feldman 1981. Forbidden oxygen lines in comets. Astron. Astrophys. 103, 154-159.

FINK, UWE, AND J. R. JoHNSON 1984. Luminosity and spatial distribution of the $[O I] 6300$ A emission in comets. Astron. J. 89, 1565-1572.

HsIEH, K. C. et al. 1987. Anisotropy of the neutral gas distribution of comet $\mathrm{P} / \mathrm{Halley}$ deduced from NGE/Vega I measurements. Astron. Astrophys. 187, 275-379.

Johnson, J. R., U. FinK, AND S. M. LARSON 1984. Charge coupled device (CCD) spectroscopy of comets: Tuttle, Stephan-Oterma, Brooks 2, and Bowell. Icarus 60, 351-372.

KelleR, H. U. et al. 1987. Comet P/Halley's nucleus and its activity. Astron. Astrophys. 187, 807-823.

KonNo, I., AND S. WYCKOFF 1988. Atomic and molecular abundances in comet Giacobini-Zinner. COSPAR XXVII. 
Krasnopolvsky, V. A., A. YU. TKChuk, G. Moreels, AND M. GogosHEV 1987. Water vapor and hydroxyl distributions in the inner coma of comet Halley measured by the VEGA 2 three-channel spectrometer TKS. In Proceedings of the Symposium on the Diversity and Similarity of Comets, ESA SP 278, 185-190.

Larson, H. P., D. S. Davis, M. J. Mumma, and H. A. Weaver 1986. Velocity-resolved observations of water in comet Halley. Astrophys. J. 273, 381-396.

LAtham, D. W. 1985. Digital stellar speedometry. In Stellar Radial Velocities, pp. 21-34. IAU Colloquium No. 88, L. Davis Press, Schenectady, New York.

Magee-Sauer, K., F. L. Roesler, F. Scherb, and J. Harlander 1989. Spatial distribution of $\mathrm{O}\left({ }^{1} \mathrm{D}\right)$ from Comet Halley. Icarus 76, 88-99.

Malaise, D. J. 1970. Collisional effects in cometary atmospheres. I. Model atmospheres and synthetic spectra. Astron. Astrophys. 5, 209-227.

SCHLEICHER, D. G. 1983. The fluorescence of cometary OH and CN. Ph.D. thesis. University of Maryland, College Park, MD.

SChleicher, D. G., AND R. L. Mill.is 1989. Revised scale lengths for cometary NH. Astrophys. J. 94, 1008-1016.
Schloerb, F. P., M. L. Claussen, and L. Tacconi-Garman 1987. Models of cometary emission in the 18-cm OH observations of comet P/Halley. Astron. Astrophys. 187, 469-474.

SPINRAD, H. 1982. Observations of the red auroral oxygen lines in nine comets. Pub. Astron. Soc. Pac. 94, 1008-1016.

Tacconi-Garman, L. 1989. Kinematic models of cometary comae. Ph.D. thesis, University of Massachusetts, Amherst, MA.

van Dischoeck, E. F., AND A. Dalgarno 1984. The dissociation of $\mathrm{OH}$ and $\mathrm{OD}$ in comets by solar radiation. Icarus 59, 305-313.

Weaver, H. A., M. J. Mumma, and H. P. Larson 1987. Infrared investigation of water in comet $\mathrm{P} /$ Halley. Astron. Astrophys. 187, 411-418.

WyATT, W. F. 1985. The CFA system for digital correlations. In Stellar Radial Velocities, pp. 132-129. IAU Colloquium No. 88, L. Davis Press, Schenectady, New York.

Wyckoff, S., S. Tegler, AND P. A. Wehinger 1988. Abundances in comet Halley at the time of the spacecraft encounters. Astrophys. $J$. 325, 927-938.

WoszczYK, A. 1962. Le Spectre du radical $\mathrm{NH}_{2}$ : Son application aux spectres cométaires. Extrait des Memoires de la Societe Royale des Sciences de Liege. Coll. in $4^{\circ}$, Tome II, Fasc. 6. 